\title{
Emerging Strategies for Controlling Drug Release by Using Visible/Near IR Light
}

\section{Bio Moses and Youngjae You*}

Department of Pharmaceutical Sciences, University of Oklahoma, Oklahoma City, OK, \& Department of Chemistry and Biochemistry, University of Oklahoma, Norman, OK, USA

\begin{abstract}
Effective drug delivery systems require controlled drug release at the target cancer cell. While strategies for targeting tumors have been extensively studied, a better understanding of the necessary technology for controlling the spatiotemporal release of a drug is still needed. It has been established that the use of light can be a unique tool for controlling drug release. While UV light can be used for the release of biologically active, caged (deactivated) compounds, clinical application is restricted because of its limited ability to penetrate tissues as well as its cytotoxicity. Recently, the use of both tissue-penetrable visible and near IR have shown promise to overcome these limitations. In this short review, we introduce new smart strategies to convert such low energy light to a tissue-penetrable stimulus for both actively and remotely controlling drug release.
\end{abstract}

Keywords: Visible/Near IR; Controlled drug release; Singlet oxygen; Photodynamic; Photothermal; Upconversion nanoparticles

\section{Introduction}

The limited selectivity of anticancer drugs toward cancer cells makes the occurrence of systemic side effects a major factor that deters their use. A recent approach to minimize these systemic effects from chemotherapy has been to develop effective delivery systems that have the ability to control the location, quantity, and time of the release of the active drug [1]. This can be achieved by specifically delivering enough of a drug in its inactive form to the site of the tumor and then use a stimulus to release the active drug locally in/around the tumor. These drugs can be chemically modified to be inactive (prodrugs), either by being physically entrapped in delivery particles (e.g., liposomes, polymer micelles, dendrimers, or albumin), or a combination of both. To improve drug delivery, the delivery vehicle can be functionalized with a targeting vector, such as an antibody, small ligands, or aptamers (active targeting). They can also be made on a nanoscale (passive targeting using enhanced permeability and retention (EPR) effects. Once localized in the tumor, the delivery systems need to be activated to release free drugs capable of interacting with their targets. The unique biological signatures of cancer cells or tumor tissues have been explored as potential activation switches [2], such as acidic $\mathrm{pH}$ (tumor tissue/ lysosomal pH) [3,4], heat [5,6], enzymes [7], and redox potentials [8]. Recently, a wide range of electromagnetic wavessuch as light (UV, visible and near IR (NIR) light) [9], microwaves [10,11], and radios wave [12] have been proposed to control drug release. While the internal stimuli are dependent on the characteristics of the biological systems, these electromagenic waves are independent of the biological systems and can be actively and externally manipulated. The availability of light sources, light delivery methods (fiber optics), and light-absorbing materials has focused attention on the use of visible and NIR light for activating prodrugs.

UV and short visible $(<400 \mathrm{~nm})$ light can be used as an external stimulus for drug release from various delivery vehicles [13]. While such light has also been extensively used with caged compounds for spatio-temporal control of biological processes [14], it has been restricted to thin objects such as cultured monolayer cells and surface of skin, because of its limited tissue penetration ability $[15,16]$. The use of longer visible and NIR (between 650-1000 nm) light becomes attractive for use with in vivo applications because of its ability to reach deeper tissues. However, the low energy of this light poses a different problem; such light cannot directly initiate the cleavage reactions of the chemical bond (linker) that is often required for releasing the drugs. Recently, three very interesting approaches have been proposed and demonstrated to overcome this problem. These approaches involve using heat-sensitive materials in photothermal release, singlet oxygen (SO)-cleavable linkers in photodynamic release, or drug release via frequency upconversion process (Figure 1) [17].

\section{Photothermal Release}

NIR light-induced photothermal release has been successfully applied to drug delivery. To control drug release, it requires two critical components: a photonic energy converter and a heat-sensitive material. This mechanism involves two steps: (1) the photonic energy converter absorbs NIR light and transform it into heat and (2) the heat energy collapses heat-sensitive hydrogels or expands the volume of particles, resulting in drug release from the vehicle. The NIR photothermal release of anticancer drugs can be combined with photothermal therapy to improve the pharmacological effects [18-23]. Because there is a non-

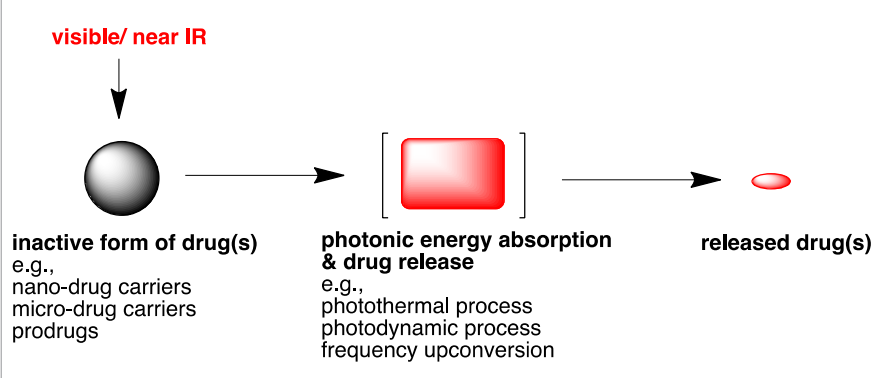

Figure 1: Three emerging strategies for visible/near IR-controlled drug release.

*Corresponding author: Youngjae You, Department of Pharmaceutical Sciences, University of Oklahoma, Oklahoma City, OK, \& Department of Chemistry and Biochemistry, University of Oklahoma, Norman, OK, USA, E-mail: youngjae-you@ouhsc.edu

Received April 26, 2013; Accepted May 22, 2013; Published May 24, 2013

Citation: Moses B, You Y (2013) Emerging Strategies for Controlling Drug Release by Using Visible/Near IR Light. Med chem 3: 192-198. doi:10.4172/2161 0444.1000138

Copyright: $\odot 2013$ Moses B, et al. This is an open-access article distributed under the terms of the Creative Commons Attribution License, which permits unrestricted use, distribution, and reproduction in any medium, provided the original author and source are credited. 
uniform distribution of both heat and the heat generators, the use of photothermal therapy may result in an incomplete tumor ablation [24]. Thus, the combination of photothermal therapy with photothermal release to localize chemotherapy could enhance treatment outcomes while minimizing the adverse side effects of chemotherapy. Sershen et al. first demonstrated that the temperature-sensitive drug delivery was possible by using polymer-nanoshell composites [25], and later significant advances have been made. Three recent examples are reviewed below:

\section{Example 1}

The use of caged compounds: Yavuz et al. [26] exposed a gold nanocage containing doxorubicin (DOX) and covered with pNIPAAmco-pAAm (poly(N-isopropylacrylamide-co-acrylamide) copolymer, which is a heat-sensitive smart polymer to NIR light (Figure 2). The nanocage absorbed the light and converted it into heat, which caused the collapse of the polymer and the released DOX. Once the NIR light irradiation was stopped, the polymer relaxed back to the original shape and consequently stopped the release of DOX.

\section{Example 2}

The use of nanorod composites: Hribar et al. demonstrated that NIR light triggered the release of the small molecule DOX from the polymer/gold nanorod composites [A6/tBA networks including gold nanorods or microspheres ( 40 um in diameter) composed of 10:20:70 wt \% A6:HEA:tBA with gold nanorods] by the photothermal effect (Figure 3) $[27,28] .{ }^{*} \mathrm{~A} 6$ : poly ( $\beta$-aminoester) $(\mathrm{PBAE})$ macromere, tBA: tert-butyl acrylate; HEA: 2-hydroxyethyl acrylate, and gold nanorod: $31 \mathrm{~nm}$ (length), $9.2 \mathrm{~nm}$ (width), and 3.6 (aspect ratio). A unique feature in this system is that the polymer transitioned from a glassy to a rubbery status at human body temperature; in turn, this enhanced the release of DOX. Both NIR-triggered and stepwise drug release was demonstrated.

\section{Example 3}

The use of heat to cause the expansion of water bubbles in microspheres (MSs) or liposomes to release of the drugs: You et al. used NIR light to modulate the release of the anticancer drug paclitaxel (PTX) from hollow gold nanospheres (HAuNSs), which was the

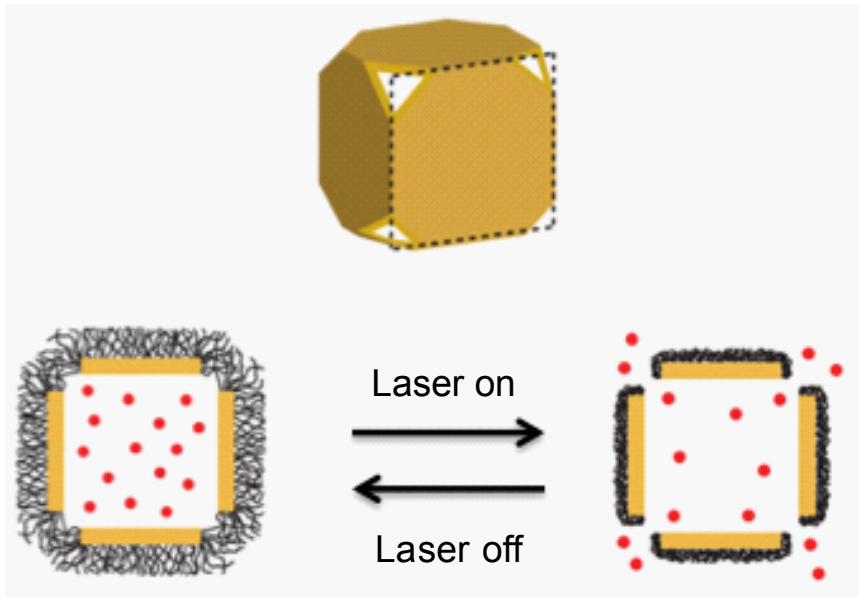

Figure 2: Schematic illustration of the NIR-controlled drug release from the polymer coated-gold nanocage. Adapted with permission from Macmillan Publishers Ltd: Nature Materials [26]. Copyright 2009.

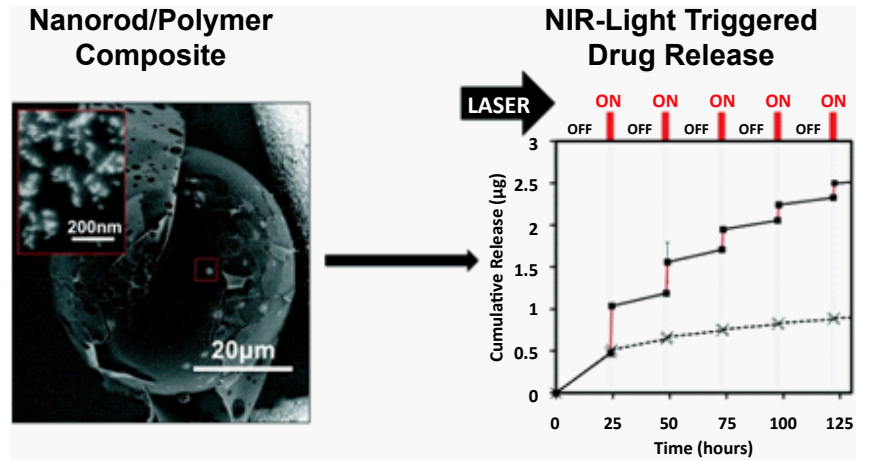

Figure 3: Backscattered micrograph image of microspheres containing gold nanorods and cumulative doxorubicin release from the microspheres. Reprinted with permission from the American Chemical Society [28]. Copyright 2011.

photonic energy converter and contained in PLGA [poly(lactide-coglycolide)] MSs (Figure 4) [19]. NIR irradiation caused the release of PTX. When the irradiation was switched off, PTX release was stopped. The local effect of the released drugs was demonstrated in both an in vivo model and in cultured cells. Liposome-nanoparticle composites have also been used for NIR-triggered release of drugs by $\mathrm{Wu}$ et al. $[29,30]$ and Volodkin et al. [31]. It was suggested that the release of drugs was caused by disruption of liposome membrane by transient vapor bubbles.

HAuNSs can also be used for cargo delivery and a photonic energy converter without a smart polymer. You et al. [21] generated DOXloaded HAuNSs that were coated with polyethylene glycol (PEG) to improve physical stability. Treating both the human breast cancer cell line MDA-MB-231 and the human ovarian cancer cell line A2780 with the combination of photothermal and chemotherapy treatment using these DOX-loaded HAuNSs resulted in an enhanced cytotoxic effects in both cell lines. To enhance the tumor targeting, they also developed the advanced HAuNSs functionalized with a EphB4 receptor-targeting peptide [22]. You et al. demonstrated a significant delay in tumor growth when NIR laser irradiation was combined with targeted T-DOX@HAuNS but not when NIR laser irradiation was combined with non-targeted DOX@HAuNS or with HAuNS. The targeted nanoparticles of T-DOX@HAuNS showed higher uptake than the nontargeted nanoparticles DOX@HAuNS in the EphB4-positive cancer cells (A2780 cells). T-DOX@HAuNs plus irradiation also showed an improved antitumor effect than DOX-@HAuNS plus irradiation in the animal model (Hey tumors).

Mesoporous silica supports were also used as a cargo/storage for NIR-triggered drug release [32]. The release mechanism in this report was unique since it was the cleavage of boronic ester bond (Figure 5). Gold nanoparticles blocked the pores of silica supports through the use of boronic ester bonds that can be cleaved by heat. The boronic ester bonds could also be hydrolyzed by $\mathrm{pH} 3$.

\section{Photodynamic Release}

The use of visible and NIR can also control drug release via photodynamic release in which low energy lightis used to generate reactive oxygen species from the combination of light, a photosensitizer (PS), and oxygen. When used to treat various diseases, this process is termed photodynamic therapy (PDT) [33]. Berg et al. developed photochemical internalization (PCI), a novel method to release drugs in endocytic vesicles to enhance the therapeutic effects (Figure 6) [34]. PCI is now a well-established method and has been applied to enhance the delivery various therapeutic molecules [35-37]. 
Citation: Moses B, You Y (2013) Emerging Strategies for Controlling Drug Release by Using Visible/Near IR Light. Med chem 3: $192-198$. doi:10.4172/2161-0444.1000138

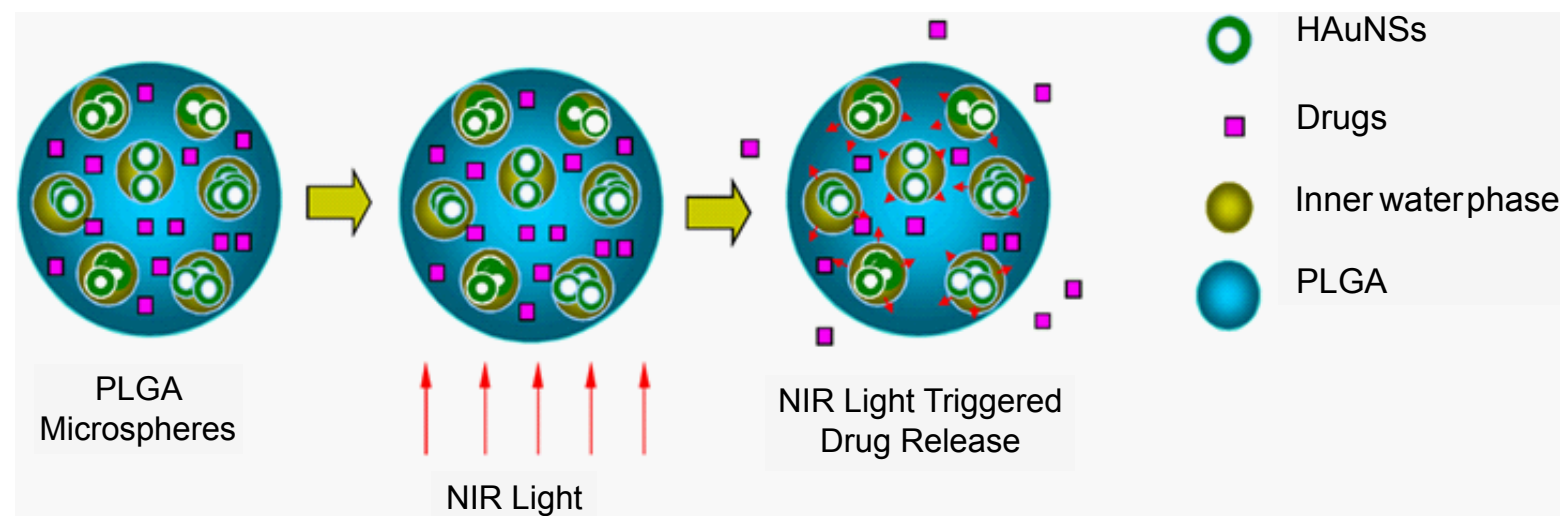

Figure 4: Hypothetical structure of PTX/HAuNS-MS and proposed mechanism of NIR-triggered drug release from the microspheres. PTX is dispersed uniformly in the matrix of PLGA polymer, whereas HAuNS are primarily dispersed in the water phase within the microspheres.Adapted from [19]. Copyright 2010 Wiley-VCH Verlag $\mathrm{GmbH} \& \mathrm{Co}$.
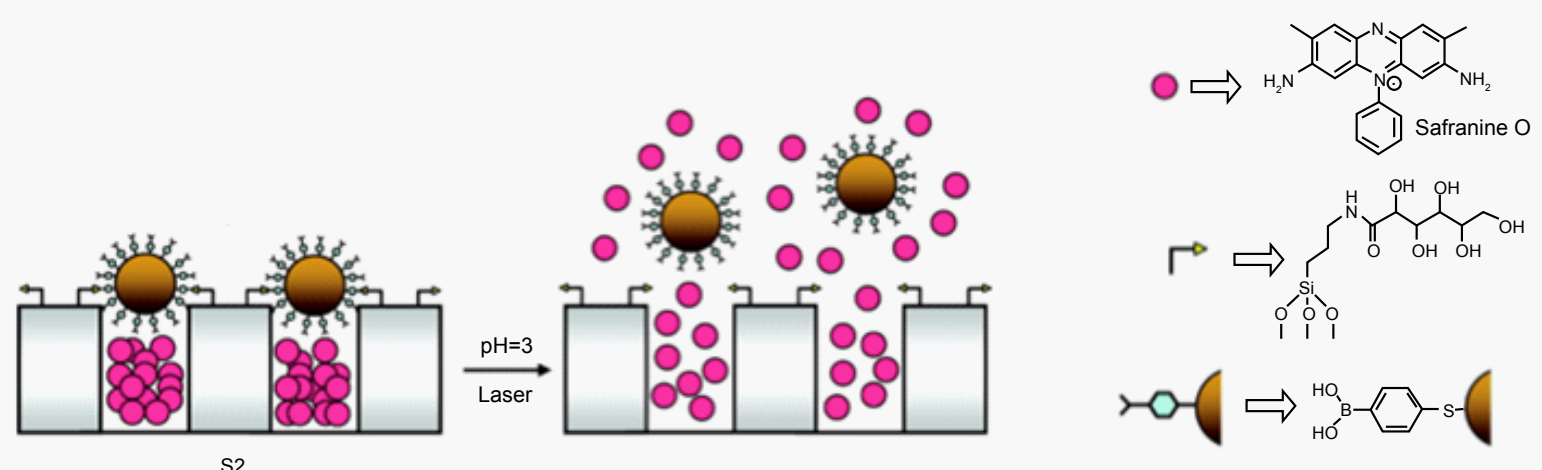

Figure 5: The pH and Laser Light Triggered Release of the Entrapped Guest (the Dye Safranine O).Reprinted with permission from the American Chemical Society [32]. Copyright 2009.

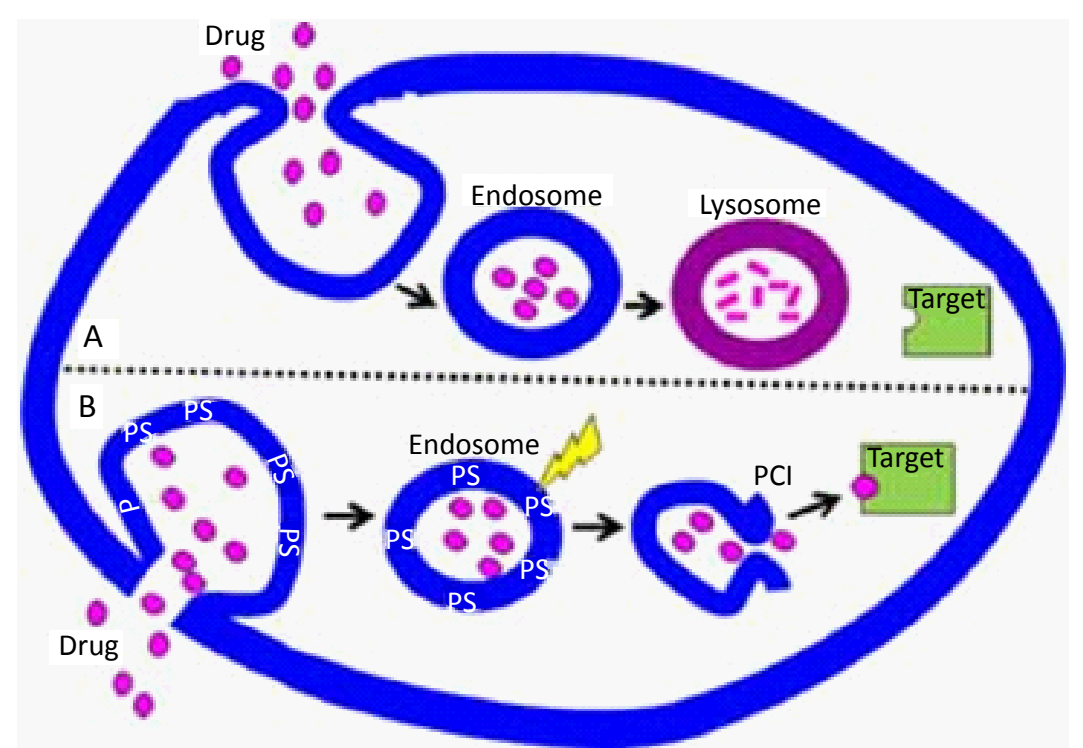

Figure 6: Schematic illustration of PCI. [38] A. No PCI: Drugs taken up through endocytosis are transported to lysosomes where they are degraded before they have exerted their action. B. PCI: PSs are co-administered with drugs and accumulate in endosomes and lysosomes. Light exposure causes rupture of the endo/lysosomal membrane and releases the drugs into the cytosol where the drugs can interact with their targets. Reprinted with permission from the [34]. Copyright $\odot$ 2011 WileyLiss, Inc. 
Citation: Moses B, You Y (2013) Emerging Strategies for Controlling Drug Release by Using Visible/Near IR Light. Med chem 3: 192-198. doi:10.4172/2161-0444.1000138

While PCIuses random oxidation of endo/lysosomal membranes to release the contents from the vesicles, chemically controlled approachescan also release drugs using photodynamic release. This reaction involves a novel concept that uses the unique reaction ofsinglet oxygen (SO).SO can undergo a [2+2] cycloaddition reaction with alkenes to form unstable dioxetanes that spontaneously decompose to give two carbonyl products (Scheme 1) [38].

The early work involving SO-mediated release was reported by Thompson et al. [39-42]. They demonstrated that SO could release compounds from liposomes via photooxidation of the plasmalogen vinyl ether linker. The phase transition that occurred by the cleavage of plasmenylcholine enhanced membrane fusion and leakage of intraliposomal contents (Scheme 2). However, this vinyl mono ether might not be a good SO-cleavable linker because the Ene-reaction of SO with a-proton of the alkene could possibly compete with the
$[2+2]$ cycloaddition reaction. They used zinc phthalocyanine, tin octabutoxyphthalocyanine and bacteriochlorophyll a in the liposomes as sensitizers, allowing longer wavelength light to be used for the activation. They used $800 \mathrm{~nm}$ to excite bacteriochlorophylla.

A similar chemistry was applied for site-specific delivery of PSs. Ruebner et al. applied an SO-mediated mechanism in the release of phthalocyanine-based PS (Scheme 3a) [43,44]. A Cyclodextrin dimer was formed by the conjugation of two $\beta$-cyclodextrins through a SOcleavable vinyl dithioether linker. The cyclodextrin dimer then binds to the phthalocyanine, and upon irradiation of light generates SO to cleave the vinyl dithioether bond, results in the release of the PS. Greer and co-worker also used an SO-cleavable linker for site-specific delivery of PSs (Scheme 3b) [45-47]. A fiber tip was conjugated to PSs through an SO-cleavable vinyl diether linker. Triplet oxygen wassupplied internally through the fiber and converted to SO at the tip of the fiber.

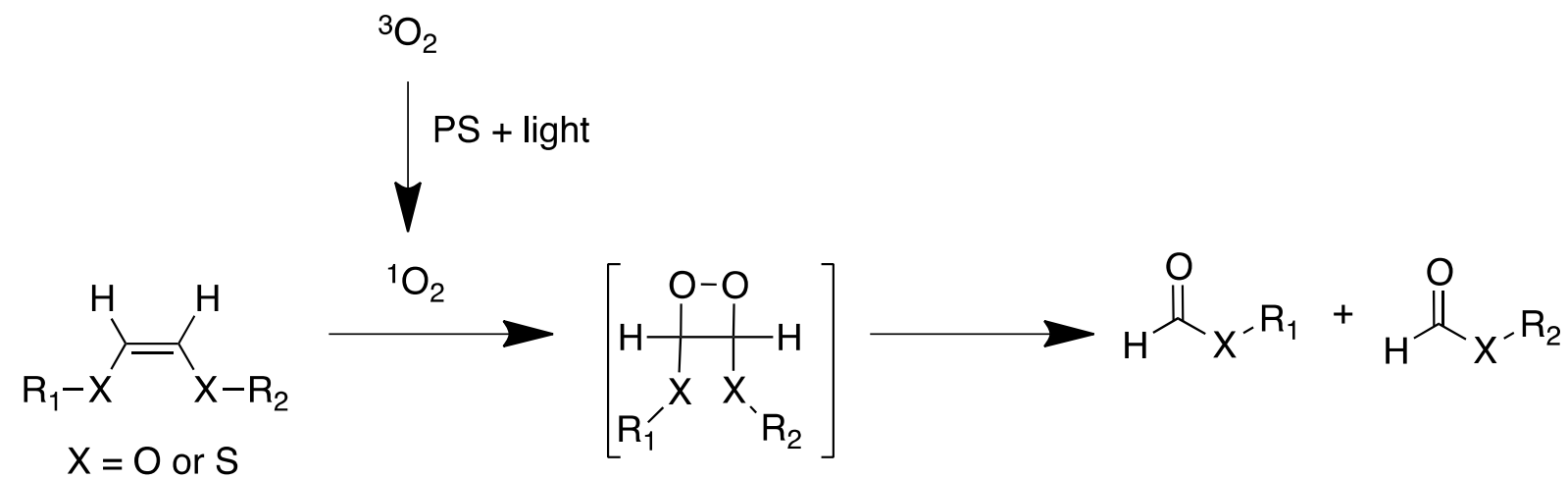

Scheme 1: Generation of SO by photodynamic effects, [2+2] cycloaddition reaction of SO with a heteroatom-substituted alkene, and subsequent cleavage of dioxetane.

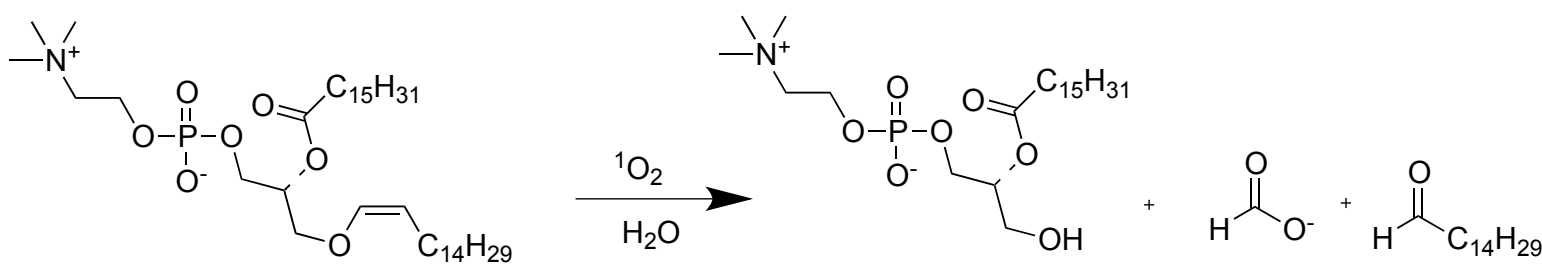

Scheme 2: Oxidation of plasmenylcholine by SO and its cleavage to the products.
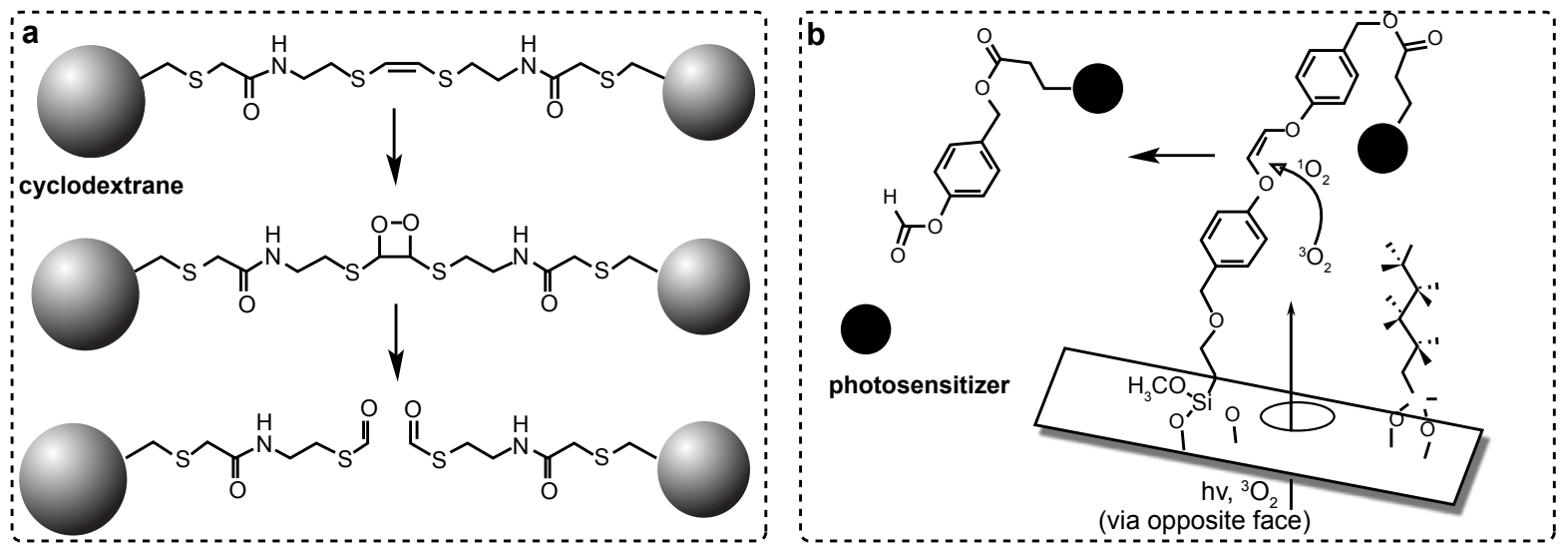

Scheme 3: a) SO-mediated cleavage of vinyl thiodiether linker and b) Release of a PS at the fiber tip supplying oxygen and light. 
Citation: Moses B, You Y (2013) Emerging Strategies for Controlling Drug Release by Using Visible/Near IR Light. Med chem 3: $192-198$. doi:10.4172/2161-0444.1000138

The SO then cleaved the vinyl diether bond to release the sensitizers. It is proposed that this fiber optics-guided delivery of oxygen and PSs could be applied to hypoxic conditions.

Activation of prodrugs by an SO-cleavable linker was demonstrated by Jiang et al. [48] and Bio et al. [49]. One of the key requirements of prodrugs is the release of the parent drug without any modification to drug structure. Thus, Jiang et al. conjugated a PS to a drug, bearing a carbonyl group through an SO-labile linker (Scheme 4a) [48]. Irradiation cleaved the SO-labile linkers releasing free drugs. Bio et al. developed a new type of SO-cleavable linker (aminoacrylate) which overcame a number of the limitations of the previous SO-cleavable linkers, such asa limited number of SO-cleavable linkers (e.g., vinyl dithioether, vinyl diether), the lack of facile synthetic approach for the cleavable linkers, andthe regeneration of the parent drug [49]. The amino acrylate linker can be synthesized by a click reaction and can be cleaved by an SO mechanismupon irradiation with a PS; this reaction is termed "click and photo-unclick chemistry" (Scheme 4b). In addition, after cleavage, the parent drug molecule can be released without any modification.

Recently, Bio et al. demonstrated the ability of this method to cleave the aminoacrylate linker and release of the drug in cancer cells [50]. The pharmacological effects of the released drugs (e.g., combretastatin A-4, CA4) were also demonstrated both in vitro and in vivo (Figure 7) [51]. A prodrug (CMP-L-CA4) of CA4 was prepared by conjugating a photosensitizer (CMP, core-modified porphyrin) and CA4 via the SO-labile linker (aminoacrylate). CMP-L-CA4 itself showed much lower activity toward tubulin polymerization and cytotoxic activity. However, once irradiated, it showed potent cytotoxic activity as close as free CA4. A more significant finding indicated that [CMP-L-CA4 + irradiation] showed significantly better antitumor effects than [CMPL-CA4 + CA4 without irradiation] and [CMP-NCL-CA4 +irradiation] (data not shown here). CMP-NCL-CA4 was an analog of CMP-L-CA4, which cannot release CA4 even after the irradiation.

a

a<smiles>[R]C([R])=COCC(C)COC(=O)S</smiles>

b

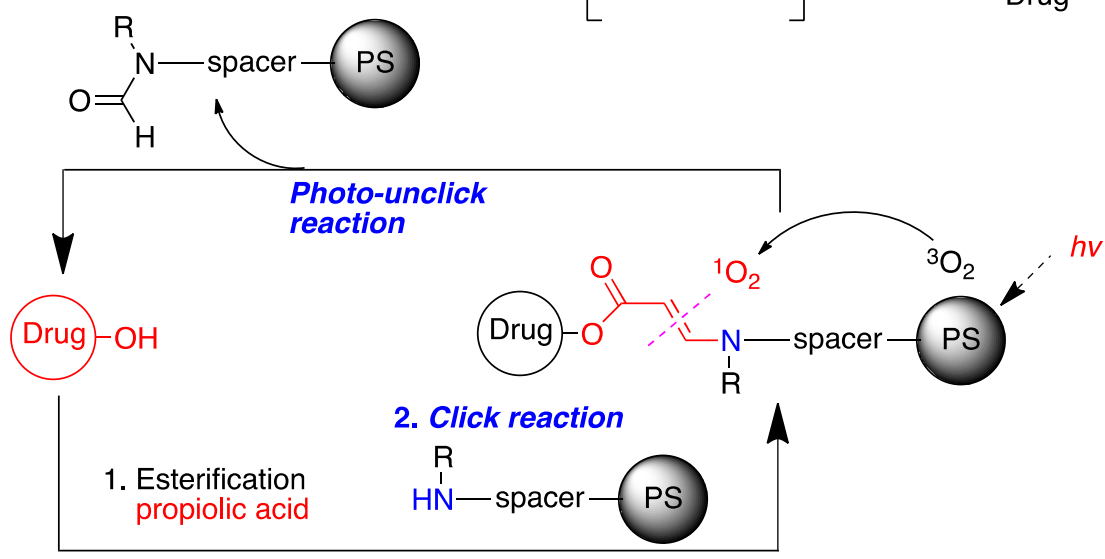

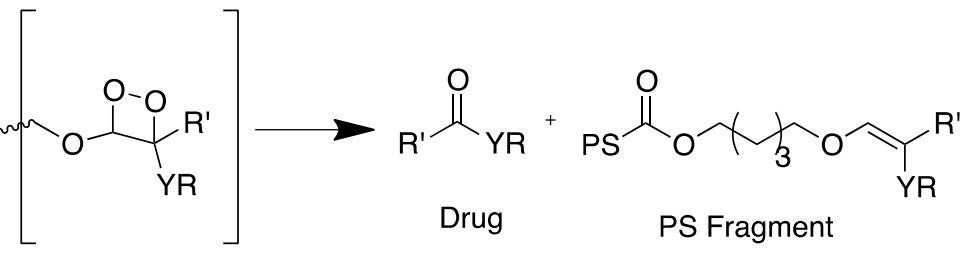

Scheme 4: a) Oxidation of the SO-labile linker of a prodrug and b) Facile synthesis, cleavage of aminoacrylate, and release of a parent drug after its oxidative cleavage. Aadapted from the [49] and reproduced with permission from the Royal Society of Chemistry (RSC). Copyright 2012.
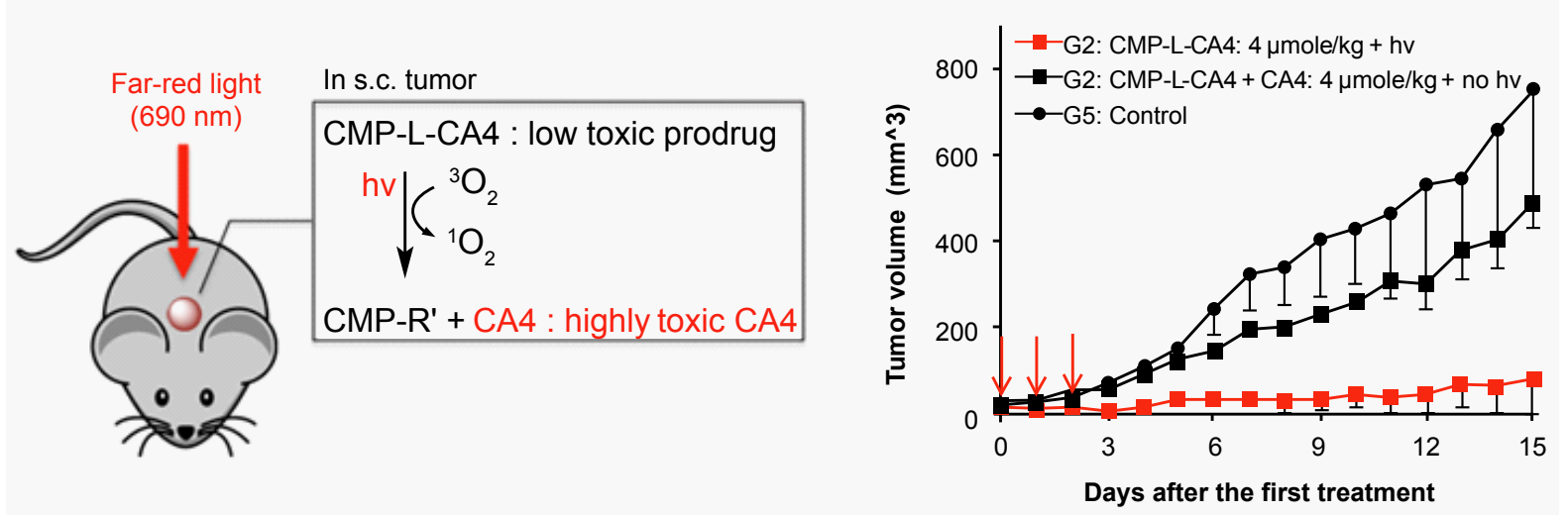

Figure 7: Far-red light-triggered release of CA4 from its prodrug (CMP-L-CA4) and the superior antitumor effect of irradiated prodrug group (G2) than non-irradiated group (G4). Reprinted with permission from the American Chemical Society [51]. Copyright 2013. 


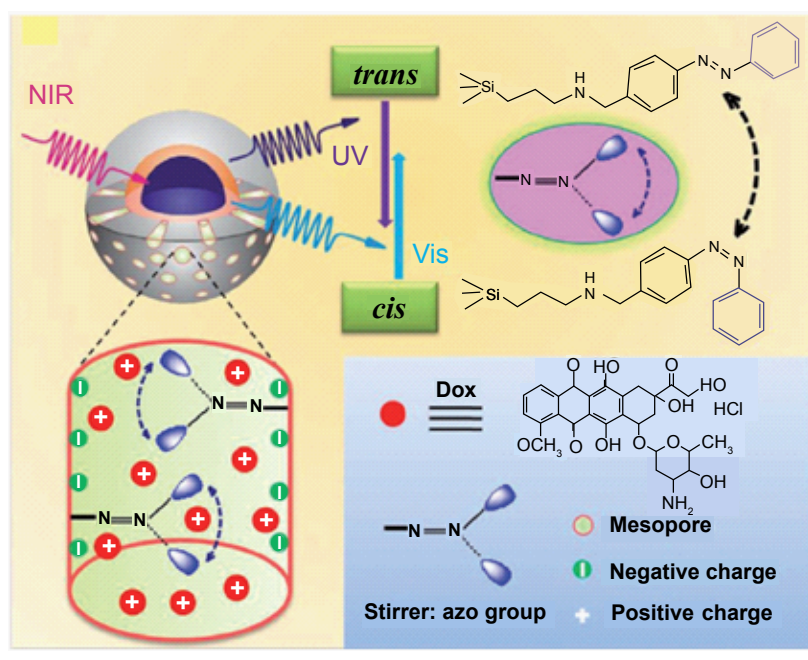

Figure 8: NIR light-triggered Dox release by the photomechanical method. Aadapted from the [54] and reproduced with permission from John Wiley \& Sons, Inc. Copyright 2013.

\section{Drug release through the NIR up conversion systems}

Most recently, two interesting strategies were proposed by taking advantage of upconverting nanoparticles (UCNPs). Nanoparticles with lanthanide ions showed a rare photonic property known as frequency upconversion. This frequency upconversion moves low energy light to higher energy light. Yang et al. [52] demonstrated this concept by using caged D-luciferin [53]. They attached caged D-luciferin on the surface of the UCNPs (Tm/Tb co-doped NaYF4). The D-luciferin was caged with 1-(2-nitrophenyl) ethyl group that can then be uncaged by UV light. They showed that the uncaged D-luciferin on the surface of UCNPs (NaYF4:TmYb) was uncaged by the irradiation with 980 $\mathrm{nm}$ light in cells and living mice. Liu et al. also employed the UPNPs (a mesoporous silica-coated UCNPs) but used a different releasing mechanism [54]. They installed "photomechanical" functional groups by using azobenzene groups that can be isomerized (cis/trans) by UV and visible light (Figure 8). Doxorubicins (DOXs) loaded in the mesoporous nanoparticles were mechanically released by NIR irradiation $(980 \mathrm{~nm})$ in solution and cells.

\section{Conclusions and Perspective}

Photothermal, photodynamic, and frequency upconversion processes represent three new strategies for releasing drugsat targets by using visible and near IR light in a spatiotemporally controlled way. Photothermal and photodynamic strategies have been successfully demonstrated both in cultured cells and in mice. On the other hand, the application of frequency upconversion is a newer concept and drug release via this method has not yet been demonstrated in animal models. It may not be necessary to directly compare their values at this point; they are at very early development stages and each method has its own pros and cons. Instead, it is both prudent and wise to find potential disease conditions effectively treated by each method.

The following points need to be considered in the development of these strategies as they aim toward clinical translation. First, materials utilized in the systems should be biocompatible: both nontoxic and biodegradable. In this aspect, the inorganic upconversion materials need to be tested for their safety. Second, the availability of light source should also be considered. At present, diode lasers seema reasonable first choice due to its affordable price and high light quality. However, available wavelengths and power levels from the diode lasers are limited, which is definitelya major factor in choosing the light absorbing materials.It is preferred to build modular delivery systems since light-absorbing material can be readily substituted to other materials absorbingavailable light sources. The photodynamic strategy is advantageous because a number of photosensitizers are available that cover a wide range in the spectral window and it can also be easily incorporated to the delivery systems. Third, the applicability and deliverability of light should be considered. Obviously, applicable disease sites for all these strategies should be organs we can externally deliver the lightsuch as skin, gastrointestinal tracts, bladder, ovary, peritoneal cavity, lung, etc. Too high intensity and dose of light used in animal studies may not be practically achievable in certain clinical settings. In general, higher intensity light was used in photo thermal and upconversion processes than in photodynamic process. Last, but perhaps the most important, these new strategies should prove to be superior than (or at lease complementary with) current methods such as surgery, radiotherapy, photodynamic and photothermal therapy.

There have been notably significant advances in the concept of visible/near IR light-controlled drug release in the past five years. This concept is no longer just in our imagination but it was provedthat we could control the drug release in tissues by using visible/near IR light. In conjunction with advances in other disciplines such as nano-technology and optical technology (light delivery and imaging systems), these smart strategies will provide important tools for both their theranostic and drug delivery applications.

\section{Acknowledgement}

The authors acknowledge the Department of Defense [Breast Cancer Research Program (W81XWH-09-1-0071)] for SO-mediated drug delivery research. Views, opinions and any endorsements by the author(s) do not reflect those of the US Army or the Department of Defense.

\section{References}

1. Kratz F, Senter $P$, Steinhagen $H$ (2012) Drug delivery in oncology from basic research to cancer therapy (3-volume series). Wiley- $\mathrm{VCH}$, Weinheim.

2. Ganta S, Devalapally H, Shahiwala A, Amiji M (2008) A review of stimuliresponsive nanocarriers for drug and gene delivery. J Control Release 126 187-204.

3. Shen Y, Tang H, Radosz M, Van Kirk E, Murdoch WJ (2008) pH-responsive nanoparticles for cancer drug delivery. Methods Mol Biol 437: 183-216.

4. Gao W, Chan JM, Farokhzad OC (2010) pH-Responsive nanoparticles for drug delivery. Mol Pharm 7: 1913-1920.

5. Schmaljohann D (2006) Thermo- and $\mathrm{pH}$-responsive polymers in drug delivery. Adv Drug Deliv Rev 58: 1655-1670.

6. Needham D, Dewhirst MW (2001) The development and testing of a new temperature-sensitive drug delivery system for the treatment of solid tumors. Adv Drug Deliv Rev 53: 285-305.

7. Meers $P$ (2001) Enzyme-activated targeting of liposomes. Adv Drug Deliv Rev 53: $265-272$.

8. Graf N, Lippard SJ (2012) Redox activation of metal-based prodrugs as a strategy for drug delivery. Adv Drug Deliv Rev 64: 993-1004.

9. Katz JS, Burdick JA (2010) Light-responsive biomaterials: development and applications. Macromol Biosci 10: 339-348.

10. Hernot S, Klibanov AL (2008) Microbubbles in ultrasound-triggered drug and gene delivery. Adv Drug Deliv Rev 60: 1153-1166.

11. Derfus AM, von Maltzahn G, Harris TJ, Duza T, Vecchio KS, et al. (2007) Remotely triggered release from magnetic nanoparticles. Adv Mater 19: 39323936.

12. Timko BP, Dvir T, Kohane DS (2010) Remotely triggerable drug delivery systems. Adv Mater 22: 4925-4943 
Citation: Moses B, You Y (2013) Emerging Strategies for Controlling Drug Release by Using Visible/Near IR Light. Med chem 3: 192-198. doi:10.4172/2161-0444.1000138

13. Alvarez-Lorenzo C, Bromberg L, Concheiro A (2009) Light-sensitive intelligent drug delivery systems. Photochem Photobiol 85: 848-860.

14. Lee HM, Larson DR, Lawrence DS (2009) Illuminating the chemistry of life: design, synthesis, and applications of "caged" and related photoresponsive compounds. ACS Chem Biol 4: 409-427.

15. Juzenas P, Juzeniene A, Kaalhus O, lani V, Moan J (2002) Noninvasive fluorescence excitation spectroscopy during application of 5 -aminolevulinic acid in vivo. Photochem Photobiol Sci 1: 745-748.

16. Meinhardt M, Krebs R, Anders A, Heinrich U, Tronnier H (2008) Wavelengthdependent penetration depths of ultraviolet radiation in human skin. J Biomed Opt 13: 044030 .

17. Rai P, Mallidi S, Zheng X, Rahmanzadeh R, Mir Y, et al. (2010) Development and applications of photo-triggered theranostic agents. Adv Drug Deliv Rev 62 : 1094-1124.

18. Park H, Yang J, Lee J, Haam S, Choi IH, et al. (2009) Multifunctiona nanoparticles for combined doxorubicin and photothermal treatments. ACS Nano 3: 2919-2926.

19. You J, Shao R, Wei X, Gupta S, Li C (2010) Near-infrared light triggers release of Paclitaxel from biodegradable microspheres: photothermal effect and enhanced antitumor activity. Small 6: 1022-1031.

20. Park JH, von Maltzahn G, Xu MJ, Fogal V, Kotamraju VR, et al. (2010) Cooperative nanomaterial system to sensitize, target, and treat tumors. Proc Natl Acad Sci USA 107: 981-986.

21. You J, Zhang G, Li C (2010) Exceptionally high payload of doxorubicin in hollow gold nanospheres for near-infrared light-triggered drug release. ACS Nano 4: 1033-1041.

22. You J, Zhang R, Xiong C, Zhong M, Melancon M, et al. (2012) Effective photothermal chemotherapy using doxorubicin-loaded gold nanospheres that target EphB4 receptors in tumors. Cancer Res 72: 4777-4786.

23. You J, Zhang R, Zhang G, Zhong M, Liu Y, et al. (2012) Photothermalchemotherapy with doxorubicin-loaded hollow gold nanospheres: A platform for near-infrared light-trigged drug release. J Control Release 158: 319-328.

24. Ren F, Bhana S, Norman DD, Johnson J, Xu L, et al. (2013) Gold nanorods carrying paclitaxel for photothermal-chemotherapy of cancer. Bioconjug Chem 24: $376-386$

25. Sershen SR, Westcott SL, Halas NJ, West JL (2000) Temperature-sensitive polymer-nanoshell composites for photothermally modulated drug delivery. $J$ Biomed Mater Res 51: 293-298.

26. Yavuz MS, Cheng Y, Chen J, Cobley CM, Zhang Q, et al. (2009) Gold nanocages covered by smart polymers for controlled release with near-infrared light. Nat Mater 8: 935-939.

27. Hribar KC, Metter RB, Ifkovits JL, Troxler T, Burdick JA (2009) Light-induced temperature transitions in biodegradable polymer and nanorod composites. Small 5: 1830-1834.

28. Hribar KC, Lee MH, Lee D, Burdick JA (2011) Enhanced release of smal molecules from near-infrared light responsive polymer-nanorod composites. ACS Nano 5: 2948-2956.

29. Wu G, Mikhailovsky A, Khant HA, Fu C, Chiu W, et al. (2008) Remotely triggered liposome release by near-infrared light absorption via hollow gold nanoshells. J Am Chem Soc 130: 8175-8177.

30. Wu G, Mikhailovsky A, Khant HA, Zasadzinski JA (2009) Chapter 14 Synthesis, characterization, and optical response of gold nanoshells used to trigger release from liposomes. Methods Enzymol 464: 279-307.

31. Volodkin DV, Skirtach AG, Mohwald H (2009) Near-IR remote release from assemblies of liposomes and nanoparticles. Angew Chem Int Ed Engl 48: 1807-1809.

32. Aznar E, Marcos MD, Martinez-MÃnez R, Sancenon F, Soto J, et al. (2009) $\mathrm{pH}$ - and photo-switched release of guest molecules from mesoporous silica supports. J Am Chem Soc 131: 6833-6843.

33. Dougherty TJ, Gomer CJ, Henderson BW, Jori G, Kessel D, et al. (1998) Photodynamic therapy. J Natl Cancer Inst 90: 889-905.
34. Berg K, Selbo PK, Prasmickaite L, Tjelle TE, Sandvig K, et al. (1999) Photochemical internalization: a novel technology for delivery of macromolecules into cytosol. Cancer Res 59: 1180-1183.

35. Norum OJ, Selbo PK, Weyergang A, Giercksky KE, Berg K (2009) Photochemical internalization ( $\mathrm{PCl}$ ) in cancer therapy: from bench towards bedside medicine. J Photochem Photobiol B 96: 83-92.

36. Selbo PK, Weyergang A, HÃ gset A, Norum OJ, Berstad MB, et al (2010) Photochemical internalization provides time- and space-controlled endolysosomal escape of therapeutic molecules. J Control Release 148: 2-12.

37. Nishiyama N, Iriyama A, Jang WD, Miyata K, Itaka K, et al. (2005) Light-induced gene transfer from packaged DNA enveloped in a dendrimeric photosensitizer. Nat Mater 4: 934-941.

38. Weyergang A, Selbo PK, Berstad ME, Bostad M, Berg K (2011) Photochemical internalization of tumor-targeted protein toxins. Lasers Surg Med 43: 721-733.

39. Anderson VC, Thompson DH (1992) Triggered release of hydrophilic agents from plasmologen liposomes using visible light or acid. Biochim Biophys Acta 1109: 33-42.

40. Thompson DH, Gerasimov OV, Wheeler JJ, Rui Y, Anderson VC (1996) Triggerable plasmalogen liposomes: improvement of system efficiency. Biochim Biophys Acta 1279: 25-34.

41. Shum P, Kim JM, Thompson DH (2001) Phototriggering of liposomal drug delivery systems. Adv Drug Deliv Rev 53: 273-284.

42. Gerasimov OV, Boomer JA, Qualls MM, Thompson DH (1999) Cytosolic drug delivery using $\mathrm{pH}$ - and light-sensitive liposomes. Adv Drug Deliv Rev 38: 317 338

43. Ruebner A, Yang Z, Leung D, Breslow R (1999) A cyclodextrin dimer with a photocleavable linker as a possible carrier for the photosensitizer in photodynamic tumor therapy. Proc Natl Acad Sci USA 96: 14692-14693.

44. Baugh SD, Yang Z, Leung DK, Wilson DM, Breslow R (2001) Cyclodextrin dimers as cleavable carriers of photodynamic sensitizers. J Am Chem Soc 123 12488-12494.

45. Zamadar M, Ghosh G, Mahendran A, Minnis M, Kruft BI, et al. (2011) Photosensitizer drug delivery via an optical fiber. J Am Chem Soc 133: 7882 7891.

46. Mahendran A, Kopkalli Y, Ghosh G, Ghogare A, Minnis M, et al. (2011) A handheld fiber-optic implement for the site-specific delivery of photosensitizer and singlet oxygen. Photochem Photobiol 87: 1330-1337.

47. Kruft BI, Greer A (2011) Photosensitization reactions in vitro and in vivo. Photochem Photobiol 87: 1204-1213.

48. Jiang MY, Dolphin D (2008) Site-specific prodrug release using visible light. $J$ Am Chem Soc 130: 4236-4237.

49. Bio M, Nkepang G, You Y (2012) Click and photo-unclick chemistry of aminoacrylate for visible light-triggered drug release. Chem Commun (Camb) 48: 6517-6519.

50. Hossion AML, Bio M, Nkepang G, Awuah SG, You Y (2013) Visible Light Controlled Release of Anticancer Drug through Double Activation of Prodrug ACS Med Chem Lett 4: 124-127.

51. Bio M, Rajaputra P, Nkepang G, Awuah SG, Hossion AM, et al. (2013) SiteSpecific and Far-Red-Light-Activatable Prodrug of Combretastatin A-4 Using Photo-Unclick Chemistry. J Med Chem

52. Chen G, Yang C, Prasad PN (2013) Nanophotonics and Nanochemistry: Controlling the Excitation Dynamics for Frequency Up- and Down-Conversion in Lanthanide-Doped Nanoparticles. Acc Chem Res

53. Yang $Y$, Shao $Q$, Deng $R$, Wang $C$, Teng $X$, et al. (2012) In vitro and in vivo uncaging and bioluminescence imaging by using photocaged upconversion nanoparticles. Angew Chem Int Ed Engl 51: 3125-3129.

54. Liu J, Bu W, Pan L, Shi J (2013) NIR-triggered anticancer drug delivery by upconverting nanoparticles with integrated azobenzene-modified mesoporous silica. Angew Chem Int Ed Engl 52: 4375-4379. 